\title{
Emmanuelle Labeau et Pierre Larrivée (dir.), Nouveaux développements de l'imparfait
}

\section{Chiara Preite}

\section{(2) OpenEdition}

1 Journals

\section{Édition électronique}

URL : http://journals.openedition.org/studifrancesi/30983

DOI : $10.4000 /$ studifrancesi.30983

ISSN : 2421-5856

Éditeur

Rosenberg \& Sellier

\section{Édition imprimée}

Date de publication : 1 avril 2006

Pagination : 211-212

ISSN : 0039-2944

\section{Référence électronique}

Chiara Preite, «Emmanuelle Labeau et Pierre Larrivée (dir.), Nouveaux développements de l'imparfait »,

Studi Francesi [En ligne], 148 (XLX | I) | 2006, mis en ligne le 30 novembre 2015, consulté le 21 avril 2021. URL : http://journals.openedition.org/studifrancesi/30983 ; DOI : https://doi.org/10.4000/ studifrancesi.30983

Ce document a été généré automatiquement le 21 avril 2021.

\section{(c) 9 (i) $\Theta$}

Studi Francesi è distribuita con Licenza Creative Commons Attribuzione - Non commerciale - Non opere derivate 4.0 Internazionale. 


\title{
Emmanuelle Labeau et Pierre Larrivée (dir.), Nouveaux développements de l'imparfait
}

\author{
Chiara Preite
}

\section{RÉFÉRENCE}

emMANUelle LABEAU et PIERRE LARRIVÉE (dir.), Nouveaux développements de l'imparfait,

«Cahiers Chronos », n 14, Rodopi B.V., Amsterdam-New York, NY 2005, pp. 204.

1 Les contributions réunies (présentées à la conférence internationale «Nouveaux développements de l'imparfait " à Aston University en 2002) visent à expliciter les valeurs et les effets de discours manifestés par le tiroir de l'imparfait (désormais IMP), dans un but à la fois descriptif et pédagogique.

2 JACQUES BRES, L'imparfait: l'un et/ou le multiple? A propos des imparfaits "narratif » et "d'hypothèse ", pp. 1-32.

Dans cet article Bres s'interroge sur l'articulation de l'un de la langue - l'IMP comme signifiant grammatical - et du multiple du discours - ses nombreux emplois, qui sont nommés par les grammaires sans être expliqués. L'IMP "semble" échapper aux tentatives d'explication unitaire fournies sur la base des repères traditionnelles du temps, de l'aspect et du mode. Toutefois l'auteur soutient une hypothèse monosémiste selon laquelle le multiple discursif n'est tel qu'en apparence car dans tous ses emplois l'IMP a une seule valeur en langue, il code la même instruction, et il écarte ainsi l'hypothèse opposée de polysémisation de l'unité, aussi bien que l'hypothèse simpliste qui constate la multiplicité sans l'articuler à l'unicité. Selon une perspective praxématique et en partie psychomécanique, Bres explique les valeurs de l'IMP narratif et d'hypothèse - qui pourraient faire l'objet de critiques - par les différences modales, aspectuelles et temporelles de représentation du temps impliqué par le procès.

CO VET, L'imparfait : emploi anaphorique et emplois non anaphoriques, pp. 33-44. 
L'auteur considère que l'IMP est un « présent dans le passé » et que le système temporel se construit à partir d'une "actualité présente » et d'une " actualité passée » : ainsi le présent est déictique car il fait référence au moment de la parole (S), alors que l'IMP est anaphorique et fait référence à un antécédent temporel donné par la phrase $\left(\mathrm{S}^{\prime}\right)$. Cela implique la nécessité de prendre en compte le système verbal dans son intégralité expression d'un système temporel du français qui n'est pas ternaire mais binaire selon Vet - afin d'expliquer les rapports que l'IMP entretient avec les autres formes du système. En se basant sur ces présupposés, l'auteur montre que les définitions traditionnelles de l'IMP en termes de durée et d'aspect sécant sont erronées ou incomplètes. Ce tiroir est déterminé par son caractère anaphorique et la perte de cette valeur donne lieu à certaines emplois, tels que l'IMP pittoresque, l'IMP dans les complétives enchâssées sous un verbe de perception et l'IMP dans les phrases conditionnelles introduites par $s i$, où l'accès à un antécédent temporel précédemment introduit dans le discours s'avère être bloqué. Dans ces cas la valeur aspectuelle de la phrase est déterminée par le mode d'action.

PATRICK CAUDAL, CARL VETTERS, Que l'imparfait n'est pas (encore) un prétérit, pp. 45-77.

7 Dans cette contribution Caudal et Vetters essayent d'appuyer une approche diachronique de l'IMP à un traitement formel. Après avoir retracé l'évolution diachronique de l'IMP à partir de l'ancien français et après avoir montré que le passage d'une description de ce tiroir en termes d'aspect «imperfectif» ou «sécant» à la reconnaissance de valeurs telles que le «narratif» ou le «perfectif» est strictement lié à l'évolution du passé simple, les deux auteurs proposent un traitement formel des phénomènes aspectuo-temporels liés à l'IMP, apte à en expliquer l'évolution et à en rendre compte en termes d'actes de force illocutoire. Le modèle explique en particulier les usages narratifs de l'IMP autrefois réservés au passé simple (PS). Cette valeur narrative, associée à la notion de transitionnalité, est devenue compatibles avec l'IMP grâce aux contextes narratifs qui marquent la progression globale d'un discours. Ainsi, le PS et l'IMP restent deux temps distincts et l'IMP garde son aspect perfectif - il n'a pas de véritable valeur de prétérit - tout en permettant un apport narratif véhiculé par le contexte : en effet la transitionnalité ne doit rien à la sémantique de ce temps mais se manifeste au niveau d'effet pragmatique.

8 EMMANUELLE LABEAU, Mon nom est narratif: imparfait narratif, pp. 79-102.

9 Inspirant son système de représentation des fonctions de l'IMP « narratif » des théories de Reichenbach, Labeau emprunte quelques traits descriptifs à plusieurs approches qui ont été proposées : temporelle, anaphorique, inactuelle, textuelle et aspectuelle. Dans le cadre d'une approche monosémique de la description des fonctions de l'IMP, l'auteur explique l'effet narratif en termes de contradiction entre la description de l'IMP et les restrictions imposées en discours, en posant une description unique de la forme IMP susceptible d'être modifiée par des éléments du contexte portant sur les composantes $\mathrm{E}$ (moment of Event) et $\mathrm{R}$ (moment of Reference). Ainsi l'auteur élargit-il la description traditionnelle de l'IMP narratif en concurrence avec le passé simple et le passé composé et essaie de présenter une liste de ses traits distinctifs : parenté avec le passé simple, aspect perfectif ou global, aspect lexical du verbe qui en influence l'emploi, présence de complément et semelfactivité. Toutefois, Labeau reconnaît que l'évolution toujours en marche de ce tiroir ne permet pas d'en systématiser les caractéristiques propres. 
11 Dans le cadre de la psychomécanique guillaumienne et de la théorie de la pertinence de Sperber \& Wilson, Saussure et Sthioul élaborent une approche selon laquelle les emplois les plus variés d'une forme verbale s'expliquent par une valeur fondamentale attachée à la langue, par une sémantique potentielle que le locuteur utilise selon les situations de communication. C'est le processus interprétatif mis en jeu par le destinataire sur la base des données contextuelles auxquelles il a accès qui peut différencier les valeurs d'une forme. Ainsi, décrire la sémantique d'un temps verbal revient à établir la procédure pragmatique appliquée par le destinataire lorsqu'il interprète la valeur d'un énoncé contenant ce temps. En ce qui concerne la sémantique de l'IMP, elle exprime une rupture de l'ancrage référentiel par rapport à la situation d'énonciation et une inclusion de ce point de repère relativement à l'événement dénoté. Pour conclure, les auteurs esquissent le dressage d'une procédure formelle (algorithmique) d'une expression telle que l'IMP. LAURENCE ROSIER, L'imparfait ventriloque ?, pp. 121-133.

Cet article est centré sur un corpus de phrases qui présentent un procès à l'IMP employé avec une troisième personne, dans le contexte de mise en scène délocutive d'une interaction, afin d'examiner les contraintes contextuelles de la personne qui favorisent le choix de l'IMP. L'analyse de Rosier part de la représentation de ce tiroir par Wilmet, qui le voit comme un sous-système (le tunc) posté à gauche - et donc ancré dans le passé - de l'actualité du sous-système du présent (le nunc) avec un aspect sécant. Parallèlement, le nunc est lié au moi, tandis que le tunc est lié au hors-moi: l'IMP déplacerait donc le moi vers la deuxième et la troisième personne, en facilitant une théâtralisation de l'énonciation que Rosier métaphorise dans l'image du «ventriloque », simulateur d'une interaction. Le but de l'auteur est de rendre compte des multiples effets de sens de ce tiroir à travers l'imbrication des temps et des personnes et à travers l'actualisation dominante tantôt du temps, tantôt de l'aspect. Ainsi elle explique les emplois «théâtraux ventriloques » de l'IMP dans le discours indirect libre (parler pour), de l'IMP hypocoristique (parler comme) et de l'IMP forain (parler à).

14 MONIQUE MONVILLE-BURSTON, JACK BURSTON, Retour à « Remise de peine » : l'imparfait, un toncal à faible marquage, pp. 135-156.

15 Faisant référence à un chapitre de « Remise de peine » de P. Modiano, dans lequel l'IMP est le temps narratif principal, Monville-Burston et Burston illustrent la multiplicité des usages de l'IMP et sa capacité à s'adapter à des contextes différents en diachronie, dans le cadre de l'hypothèse monosémiste selon laquelle les significations multiples de l'IMP s'expliquent en termes d'un invariant sémantique sous-jacent. D'une part, les auteurs explorent le trait sémantique de la distanciation, à savoir la capacité de l'IMP de présenter les événements décrits comme dissociés de l'acte de parole, en dehors de la situation d'énonciation, et de le transporter dans «l'actualité d'un autre monde ». D'autre part, ils définissent l'IMP comme «toncal faiblement marqué » (marqué par la distanciation mais neutre en termes de spatialisation), ce qui peut justifier tous les emplois assumés au fil du temps par l'IMP, mais aussi les innovations tentées par Modiano. Ce narrateur crée la sensation d'impossibilité à parvenir à une lecture définitive du texte, grâce à l'emploi de l'IMP dans des situations d'absence ou de contradiction d'indices contextuels pouvant servir de point de repère. Pour conclure, Monville-Burston et Burston introduisent la notion de «multivalence » de l'IMP, due à 
son faible marquage, qui rend compte de la richesse et de la complexité de la narration de Modiano.

ANNE-ROSINE DELBART, L'imparfait : une affaire d'instruction(s), pp. 157-173.

Delbart étudie l'IMP « caméléon » en se rangeant dans l'optique monosémiste : ce tiroir est identique à lui-même à travers toutes les réalisations discursives que le contexte permet. L'auteur s'inspire du système de Wilmet dont elle expose les grandes lignes pour parvenir à suggérer quelques illustrations personnelles, concernant deux questions auxquelles l'IMP, dans toutes ses valeurs et tous ses emplois, doit satisfaire : pourquoi le temps passé ? et pourquoi l'aspect sécant? Ensuite, Delbart élabore et propose une application didactique finalisée à aider les étudiants non francophones, et en particulier de langue maternelle non romane, à maîtriser les temps du passé en français : elle conçoit une démarche en quatre consignes ou instructions théoriques, qui sont résumées sous la forme d'un arbre de Porphyre, tout en soulignant que son travail de linguiste doit trouver application dans le travail du pédagogue.

MARTIN HOWARD, Les contextes prototypiques et marqués de l'emploi de l'imparfait par l'apprenant du français langue étrangère, pp. 175-197.

9 À la différence des autres contributions de ce volume, l'article de Howard examine la spécificité des emplois de l'IMP par l'apprenant du français langue étrangère (FLE), en identifiant ses emplois plus marqués par rapport aux emplois moins marqués, du point de vue de la difficulté que ces emplois posent aux apprenants. À cette fin l'auteur a recours aux concepts de markedness - pour identifier les emplois plus ou moins marqués de l'IMP dans des groupes d'étudiants différenciés par leurs caractéristiques extralinguistiques - et de prototype - pour identifier les caractéristiques sémantiques des contextes marquées et moins marqués. Afin de répondre à une série de questions de recherche, Howard élabore une méthode qui implique la participation des apprenants divisés en groupes à un entretien oral dans le cadre d'un projet sur l'acquisition de l'IMP en FLE. L'auteur relate les résultats obtenus en détaillant l'occurrence relative de ce tiroir par rapport à d'autres formes aspectuo-temporelles telles que le présent et le passé composé, que les apprenants utilisent en alternance. Il apparaît ainsi que l'emploi de l'IMP est influencé par les contextes que les apprenants considèrent plus favorables, comme par exemple les contextes statiques où l'emploi de l'IMP est prototypique. En revanche certains contextes marqués par exemple par la valeur fréquentative se révèlent moins favorables parce non prototypiques.

PIERRE LARRIVÉE, Quelques questions sur les nouveaux emplois de l'imparfait: conclusions du colloque et compte rendu de la session plénière, pp. 199-204.

Le volume se conclut sur une synthèse, rédigée par P. Larrivée et révisée par les contributeurs, des discussions et des conclusions du colloque international, qui souligne les points de consensus par rapport au traitement de certains valeurs de l'IMP et met en évidence les questions sur lesquelles ce consensus n'est pas encore acquis. 